\title{
Diseño e implementación de un sistema para determinar el impuesto predial a través del procesamiento $\mathrm{y}$ análisis de imágenes satelitales
}

\author{
Roman Alejandro Hernández-Moreno, Jessica Gabriela Azpeitia-Rodríguez, \\ Derlis Hernández-Lara, Leonardo Miguel Moreno-Villalba \\ Tecnológico Nacional de México, \\ Tecnológico de Estudios Superiores de Ecatepec, \\ Ecatepec, Estado de México, México \\ \{derlis392\}@hotmail.com, \{jessy.lucis715, shisuka.ari\}@gmail.com
}

\begin{abstract}
Resumen. Este trabajo presenta la forma de implementar recursos de análisis y procesamiento de imágenes para obtener un registro de los inmuebles no actualizados y registrados ante el registro público de la propiedad y comercio, con el fin de poder realizar un cobro justo a los contribuyentes, actualizando de manera eficiente la base de datos correspondiente. Para el sistema se toma en cuenta una referencia que permite obtener una base de bits, la cual se opera en la matriz de pixeles obtenida a partir del análisis de la imagen, después mediante un suavizado de la misma y la función Gaussiana se obtiene una imagen que permite determinar los bordes que componen una estructura o edificación, para posteriormente mediante una red neuronal analizar las líneas que conforman el edificio tomando en cuenta las variables más significativas. El problema en este tipo de sistemas es la determinación de las medidas y la toma valores erróneos durante la obtención de los espacios de estudio. Con la propuesta realizada se ha resuelto este problema a partir de imágenes satelitales y una red neuronal, generando de esta forma un cuadro de referencia encontrado a partir del estudio de la matriz de la imagen.
\end{abstract}

Palabras clave: análisis y procesamiento de imágenes, función Gaussiana, detección de bordes, redes neuronales, impuesto predial.

\section{Design and Implementation of a System to Determine the Property Tax through the Processing and Analysis of Satellite Images}

\begin{abstract}
This work presents the way to implement resources for analysis and image processing to obtain a registry of the properties not updated and registered with the public registry of the property and commerce, in order to be able to make a fair payment to the taxpayers, updating of Efficiently the corresponding database. For the system a reference is taken into account that allows to obtain a bit base, which is operated in the matrix of pixels obtained from the analysis of the image, then by smoothing it and the Gaussian function an image is obtained which allows to determine the edges that make up a structure or building, and then through a neural network analyze the lines that make up the building taking
\end{abstract}


into account the most significant variables. The problem in this type of systems is the determination of the measures and takes wrong values during the obtaining of the study spaces. With the proposal made, this problem has been solved from satellite images and a neural network, thus generating a reference chart found from the study of the image matrix.

Keywords: image analysis and processing, Gaussian function, edge detection, neural networks, property tax.

\section{Introducción}

Uno de los principales objetivos al implementar metaheurísticas en problemas de ingeniería, es el de resolver situaciones complejas y buscar soluciones factibles dentro de un intervalo definido por las cotas de diseño. Con apoyo de técnicas heurísticas como las redes neuronales, se logró encontrar las secciones que permiten obtener las características de interés para llevar a cabo el estudio de las regiones importantes de una imagen, el análisis y procesamiento digital de imágenes permite suavizar el archivo a trabajar y seccionar el área de análisis en regiones definidas como filas y columnas, lo que da como resultado una matriz de pixeles, realizando de esta manera la medición de las coordenadas del inicio y termino de la región de análisis, tomándolo como punto de partida para la creación de un cuadro de referencias a examinar. Una vez llenado este requerimiento se puede regresar a la imagen suavizada con la que se determinará por medio de la función Gaussiana los bordes altos de la imagen, encontrando así los bordes generados para las estructuras de interés.

\section{Antecedentes y trabajos previos}

En [1] se diseñó un prototipo de sistema que usa distintos tipos de técnicas de computación para realizar análisis de imágenes mediante modelos de redes neuronales, dicho sistema usa información numérica (a nivel de pixel) para realizar tareas de interpretación de imágenes, identificando la presencia de ciertas estructuras oceánicas en África e Islas Canarias.

Mientras que en [2], se desarrolla una herramienta computacional para el apoyo médico que identifique si se tiene un tumor maligno o benigno, haciendo uso del procesamiento digital de imágenes, así como de las redes neuronales artificiales.

Por otra parte, en [3] se lleva a cabo el diseño de una herramienta geoespacial que tiene la capacidad de detectar automáticamente objetos en fotografías satelitales usando técnicas de procesamiento de imagen y visión artificial.

Además, en [4] se diseñó y construyó un sistema multibiométrico para la identificación de personal mediante visión artificial de iris y huella digital, la imagen obtenida se manipula para extraer información a través de la transformada de Wavelet de Haar y/o bifurcaciones y correlación adaptiva en 2D, para su clasificación se usan redes neuronales.

De igual manera, en [5] se intenta relevar las superficies boscosas utilizando procesamiento de imágenes satelitales para su posterior clasificación y ordenamiento de los recursos forestales en tierra del fuego. 
Diseño e implementación de un sistema para determinar el impuesto predial...

Así mismo, en [6] se lleva a cabo el análisis y detección de edificios de forma automática/semiautomática sobre imágenes aéreas y satelitales mediante la obtención de umbrales haciendo uso de herramientas de cómputo.

También, en [7] se trata el diseño de un sistema automático de inspección que puede mejorar este proceso de control de calidad en las empresas textiles, empleando análisis de imágenes para eliminar las imperfecciones de las telas.

Además, en [8] se realiza la elaboración de un software de procesamiento de imágenes para los análisis metalográficos para la determinación y automatización del estimado métrico del grano y fases de ferrítico-perlíticas en aceros de bajo contenido de carbono.

Para finalizar, en [9] se llevó a cabo el desarrollo de una metodología para clasificación digital enfocada a la generación de cartografía referente a la Reserva Nacional Valdivia empleando imágenes satelitales, realizado mediante un método de clasificación supervisado y aplicando un algoritmo de máxima probabilidad.

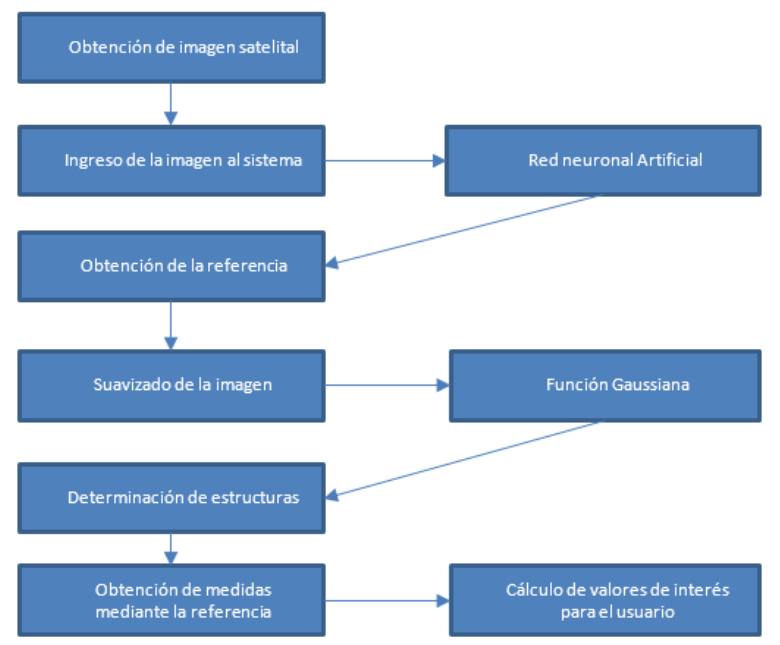

Fig. 1. Proceso considerado para el diseño del sistema

Respecto a los antecedentes mencionados, las desventajas son que los resultados obtenidos son muy ideales y difíciles de llevar a la práctica, sin embargo, esta investigación pretende contribuir a subsanar esta situación, mediante la implementación de restricciones que permitan obtener resultados factibles y que conlleven a utilizar esta metodología para diversos diseños con procesamiento de imágenes y redes neuronales en ingeniería.

\section{Metodología}

De acuerdo con el problema planteado, es posible adaptar el uso de las heurísticas para encontrar las regiones necesarias en el sistema, ya que son métodos de optimización y análisis que es posible utilizar para hallar soluciones a problemas no lineales o de complejidad analítica que son difíciles de especificar en un sistema no inteligente. 
Basado en esto, se recurre al uso de las redes neuronales artificiales para el análisis de imágenes, con lo cual se utilizan dos tipos de entrenamientos para la red utilizada en este trabajo, cómo los son aprendizaje supervisado y aprendizaje por refuerzo.

Permitiendo así, entrenar a la red de tal manera que sea capaz de llevar a cabo el análisis requerido por el sistema y entrenándose así misma constantemente con cada uso. Para este caso se analiza el sistema de acuerdo con la figura 1.

\subsection{Redes neuronales}

Para la implementación de una red neuronal artificial es necesario entender que estas están basadas en una red neuronal biológica, por lo que su elemento básico es la neurona, por lo cual se busca generar una estructura que emule esta condición. Un sistema neuronal biológico este compuesto por millones de neuronas organizadas en capas. En la emulación de dicho sistema neuronal biológico, por medio de un sistema neuronal artificial, se puede establecer una estructura jerárquica similar a la existente en el cerebro.

Para llevar a cabo el diseño del sistema es necesario tener en cuenta el funcionamiento de una red neuronal artificial, por lo cual es importante analizar la estructura y arquitectura de las neuronas. Este trabajo se basa en una arquitectura de neurona de McCulloch-Pitts, esta funciona de manera muy similar a como funciona una neurona biológica. Para ser más precisos, es una unidad de cálculo que intenta emular el comportamiento de una neurona biológica, en la figura 2 se puede apreciar la arquitectura de la neurona artificial.

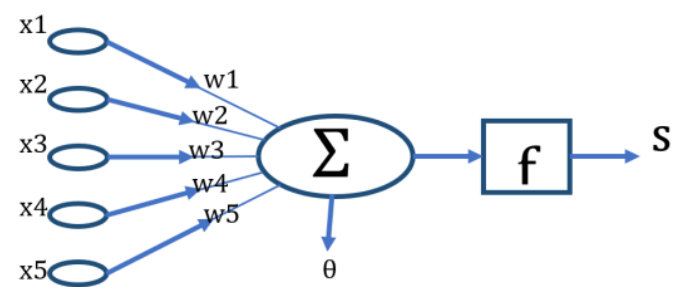

Fig. 2. Arquitectura de la neurona artificial.

Esta neurona artificial está formada por los siguientes elementos:

- $\quad \mathrm{X}$ : Conjunto de n componentes denominados vector de entradas al sistema.

- W: Conjunto de pesos sinápticos de la conexión entre la entrada y la neurona.

- $\quad \Sigma$ : Sumatoria de procesamiento de los valores de entrada.

- $\quad \theta$ : valor umbral.

- F: Función de activación que proporciona el estado de activación de la neurona en función.

- S: salida de la neurona.

Dentro de la neurona se lleva a cabo el cálculo de la siguiente ecuación (1):

$$
\sum_{i=1}^{n} w_{i} x_{i}
$$


Debiendo tomar en cuenta que la arquitectura de McCulloch-Pitts permite dos posibles resultados 1 y 0 , haciendo así necesario el comparar el resultado obtenido con el umbral de la neurona con el fin de detonar la función de activación (ecuación 2) enviando por medio del axón el resultado de la red neuronal:

$$
s_{1}\left\{1 \text { si } \sum_{i=1}^{n} \quad w_{i} x_{i} \geq \theta 0 \text { si } \sum_{i=1}^{n} \quad w_{i} x_{i}<\theta .\right.
$$

Las neuronas funcionan como procesadores de información sencillos. De manera simplista las dendritas constituyen el canal de entrada de la información, el soma es el órgano de cómputo, el axón corresponde al canal de salida, y a la vez envía información a otras neuronas. Cada neurona recibe información de aproximadamente 10000 neuronas y envía impulsos a cientos de ellas.

\subsection{Obtención de la referencia}

En esta parte del sistema se buscará la imagen satelital, que se utilizará como referencia, en este caso para el análisis y como prueba del sistema. En esta sección se toma como referencia al Tecnológico de Estudios Superiores de Ecatepec, en específico el edificio de la división de informática como ejemplo, como se muestra en las figuras 3 y 4 respectivamente. Una vez obtenida la imagen es posible notar que en la parte inferior derecha contiene una medida de referencia aproximada, que se tomará como referencia en el sistema para aproximar una unidad de medida (véase figura 5).

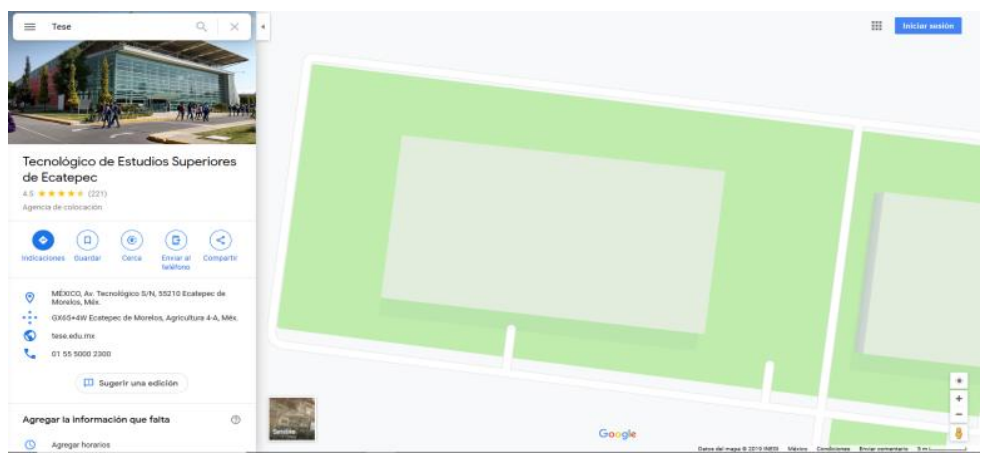

Fig. 3. Plataforma Google Maps.

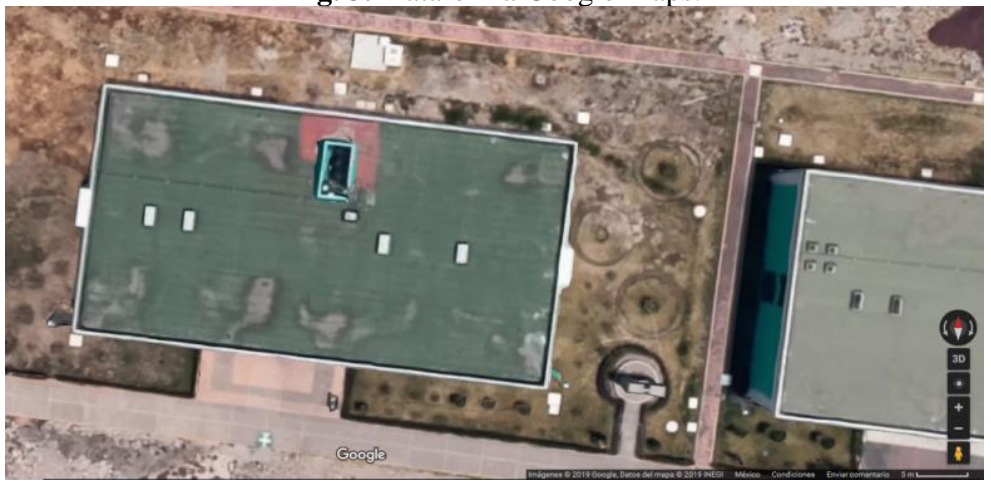

Fig. 4. Imagen satelital de prueba. 
Tomando como ejemplo la imagen obtenida, esta es ingresada por medio del asistente de exploración de archivos del sistema, donde mediante el procesamiento de esta imagen es posible detectar la posición de la referencia que se tomará como parte de los datos necesarios para el cálculo de resultados.

\section{$5 \mathrm{mLL}$}

Fig. 5. Referencia de medidas a tomar por el sistema.

Una vez obtenida la referencia, el sistema almacena el número de pixeles obtenidos de la aproximación métrica, con lo que genera una referencia visual que utiliza para una vez detectados los objetos sea posible comparar las dimensiones de cada objeto con el valor, generando una escala entre píxel por metro o número de pixeles en un metro de la misma manera en que se obtiene la referencia de la imagen satelital, en este paso por medio del procesamiento de imágenes se agrega dicha información. El resultado será algo similar a lo que se muestra la figura 6 , en donde se puede ver el archivo en la etapa de procesamiento, parte en que se agrega el referente visual para el análisis de las dimensiones de las estructuras detectadas en la fotografía.

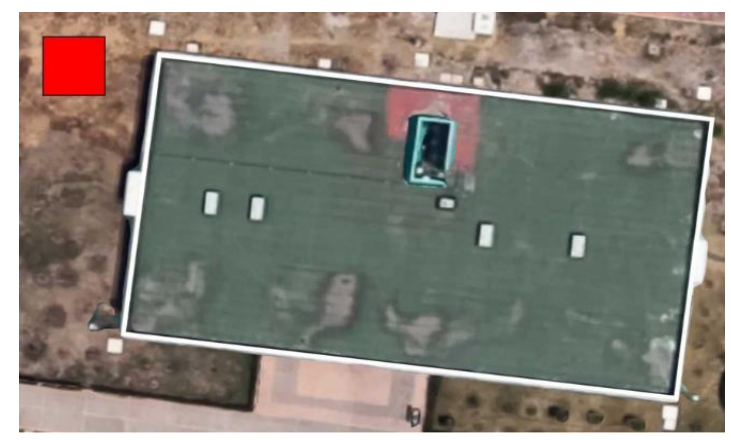

Fig. 6. Imagen satelital con referencia de medida.

La figura anterior ayuda a entender la importancia de la referencia de medida, ya que el sistema genera la referencia a través del estimado de medida entregado por la plataforma de donde se obtuvo la imagen. Basado en esto se obtendrán las unidades de medición requeridas para el sistema.

\subsection{Detección de bordes}

La detección de bordes es una técnica de análisis y procesamiento de imágenes que permite identificar objetos y aislarlos. Una vez obtenidas las salientes que conforman los bordes de una figura, es posible llevar un conteo de los objetos que se muestran en la imagen que se está utilizando como objeto de estudio, con el fin de almacenar mediante matrices las posiciones en coordenadas de cada punto encontrado como un borde.

En este caso se está haciendo uso de la llamada visión artificial, la cual es un subcampo de las matemáticas que abarca muchas técnicas que permiten llevar a cabo el tratamiento digital de imágenes. Con esta técnica se puede contar objetos específicos 
haciendo uso de la herramienta OpenCV. Durante el análisis de imágenes es preferible que exista un contraste claro entre los objetos que se están buscando y el fondo. Esto permitirá al algoritmo realizar el análisis de una manera más eficiente.

El proceso se divide en 5 fases:

1. Convertir la imagen a escala de grises

2. Filtrar la imagen para eliminar el ruido

3. Aplicar el detector de bordes

4. Buscar los contornos dentro de los bordes detectados

5. Dibujar dichos contornos

Durante la aplicación de estos pasos se obtiene una imagen cada vez más procesada, dando como resultado un conjunto de matrices que contienen las posiciones en coordenadas de cada punto encontrado como un borde. Este proceso está siendo aplicado por medio de OpenCV haciendo uso del lenguaje Python.

\section{Resultados y discusión}

El conjunto encontrado se pinta sobre la imagen original, lo que permitirá como usuarios ver los bordes detectados por el sistema representados por líneas de color rojo sobre la imagen original, se usa este color con el objetivo de que sean lo más visibles posible y que resalten tanto para el sistema como para el usuario, y con lo que al finalizar, se puede visualizar los resultados en pixeles y la cantidad de objetos que se detectaron dentro de la imagen, con lo que se genera el cálculo de las medidas en metros, como se muestra en la figura 7.

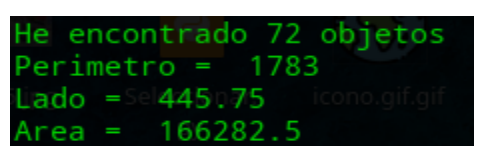

Fig. 7. Medidas en pixeles y conteo de objetos.

Para finalizar, como resultado se muestran las medidas obtenidas en metros sobrepuestas a la imagen como se observa en la figura 8, que permitirá realizar los cálculos correspondientes para encontrar los valores requeridos.

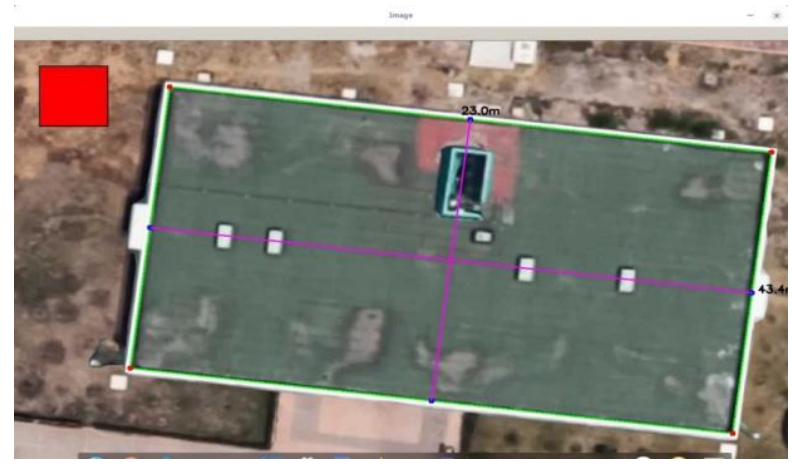

Fig. 8. Medición de objetos. 


\section{Conclusiones y trabajo futuro}

Se ha logrado desarrollar el diseño de un sistema para la determinación del impuesto predial a través del procesamiento y análisis de imágenes satelitales, utilizando métodos como las redes neuronales artificiales, análisis y procesamiento de imágenes, determinación de medidas con respecto a matrices generadas a partir de imágenes. Es posible destacar que el uso de las heurísticas en la implementación del sistema diseñado ha sido de mucha utilidad debido a la complejidad de la detección de ciertos componentes requeridos por el sistema, dentro tanto de las imágenes como de las matrices de bits utilizadas durante la detección de estructuras.

Con el fin de continuar con el avance en el desarrollo alcanzado dentro de este trabajo, se propone implementar otros algoritmos de Deep learning para la actualización automática de valores, que permitan al sistema trabajar más inteligentemente, para de esta manera lograr realizar el objetivo con mayor eficiencia y así el usuario pueda hacer uso de los resultados con base a información precisa.

Agradecimientos. Los autores agradecen al Tecnológico de Estudios Superiores de Ecatepec, en específico a la división de informática por el apoyo brindado.

\section{Referencias}

1. Torres, A.J., Cantón, M.: Detección y reconocimiento de estructuras oceánicas en imágenes AVHRR en el área de las islas canarias y NO de África mediante técnicas de computación conexionista. Revista de Teledetección, vol. 13, pp. 5-12 (2000)

2. Flores Gutiérrez, H.: Redes Neuronales Artificiales aplicadas a la detección de Cáncer de Mama. Tesis doctoral, Instituto Politécnico Nacional, México (2015)

3. Iglesias García, A.: Desarrollo de una herramienta para la detección de objetos de interés en datos multimedia georreferenciados. Tesis doctoral (2018)

4. Collado, L.: Los bosques de Tierra del Fuego. Análisis de su estratificación mediante imágenes satelitales para el. Multequina, $\mathrm{n}^{\circ}$ 10, pp. 1-15 (2001)

5. Cristina, F., Dapoto, S.H., Artola, V., Russo, C.C., Viegas, G.M.: Detección de edificios en imágenes urbanas. In: XV Congreso Argentino de Ciencias de la Computación (2009)

6. Alvarado, J.D., Alvarado F.J.: Análisis de textura en imágenes a escala de grises, utilizando patrones locales binarios (LBP). ENGI Revista Electrónica de la Facultad de Ingeniería, Vol. 1, No. 1 (2015).

7. Llulluna, L., Fredy, R.: Procesamiento de imágenes mediante software libre python para el análisis metalográfico en aceros de bajo contenido de carbono. Quito, pp. 60-79 (2014)

8. Segura, R., Trincado, G.: Cartografía digital de la Reserva Nacional Valdivia a partir de imágenes satelitales Landsat TM. Bosque (Valdivia), vol. 24, nº 2, pp. 43-52 (2003) 